\title{
Improving Performance and Retention of Engineering Graduate Students through Motivation and Identity Formation
}

\author{
Dr. Cheryl Cass, North Carolina State University
}

Cheryl Cass is a teaching assistant professor in the Department of Materials Science and Engineering at North Carolina State University where she has served as the Director of Undergraduate Programs since 2011. Her research focuses on the intersection of science and engineering identity in post-secondary and graduate level programs.

\section{Dr. Adam Kirn, University of Nevada, Reno}

Adam Kirn is an Assistant Professor of Engineering Education at University of Nevada, Reno. His research focuses on the interactions between engineering cultures, student motivation, and their learning experiences. His projects involve the study of student perceptions, beliefs and attitudes towards becoming engineers, their problem solving processes, and cultural fit. His education includes a B.S. in Biomedical Engineering from Rose-Hulman Institute of Technology, a M.S. in Bioengineering and Ph.D. in Engineering and Science Education from Clemson University.

\section{Mrs. Marissa A Tsugawa-Nieves, University of Nevada, Reno}

Marissa Tsugawa is a graduate research assistant studying at the University of Nevada, Reno in the PRiDE Research Group. She is currently working towards a Ph.D. in Engineering Education and expects to graduate May of 2019. Her research interests include student development of identity and motivation in graduate engineering environments and understanding creativity in engineering design processes.

\section{Heather Perkins, North Carolina State University}

Heather entered the Applied Social and Community Psychology program in the fall of 2014, after completing her Bachelor of Science in Psychology from the University of Cincinnati. She has participated in various research projects examining the interaction between stereotypes and science interest and confidence, their influence upon womens' performance in school and the workplace, and their presence in the media and consequences for viewers. Her primary research interest is science identity, STEM education, and participation in online communities.

Ms. Jessica Nicole Chestnut, North Carolina State University

Mr. Daniel Eamon Briggs, North Carolina State University

I am graduating from North Carolina State with a BS in Statistics in May 2017. In September 2017 I will be attending Harvard TH Chan School of Public Health to pursue a MS in Biostatistics.

\section{Blanca Miller, University of Nevada, Reno}




\section{Improving Performance and Retention of Engineering Graduate Students through Motivation and Identity Formation}

\section{Introduction}

The goal of this project is to improve the understanding of how graduate student experiences influence engineering identity formation ${ }^{1}$ and goal setting processes. ${ }^{2}$ Engineering identity and student goal setting processes have been shown to be important factors for undergraduate student participation in engineering communities of practice but have not been applied to engineering graduate communities. Through a mixed methods approach, this study will investigate graduate student motivational goal setting and identity formation to answer the following research questions:

RQ1: What are the identity and motivation profiles of engineering doctoral students, which are based on previous academic and research experiences in STEM?

\section{RQ2: How does the STEM community influence identity formation and motivational goal setting processes of engineering doctoral students?}

\section{RQ3: How do these processes related to identity formation and motivation influence engineering graduate student retention, productivity, and pursuit of doctoral level engineering careers?}

Results of this study will inform programmatic decisions in engineering graduate programs and facilitate targeted interventions that promote motivation and identity development of students. This work also aims to shape graduate education best practices for recruitment, retention, and training in engineering disciplines.

\section{Broad Methodological Plan}

In the initial, qualitative phase (Phase 1) of the project, we recruited $\mathrm{Ph} . \mathrm{D}$. students in engineering programs to participate in focus groups and interviews about their graduate-level academic and research experiences. Interview transcripts were analyzed using an Interpretative Phenomenological Analysis (IPA) approach. ${ }^{3}$ The goal of the analysis was to understand the lived engineering experiences of the students and the meaning found in these experiences within the context of the project's focus on identity and motivation. An IPA approach allows connections between graduate experiences and student affective domain traits that are complex and may not be readily visible to students. In addition to describing the experiences of students and identifying key themes and features of these experiences, results from IPA analysis will inform item development in a quantitative instrument. This survey (Phase 2) will be deployed to 5,000 graduate students in engineering programs; among other quantitative analyses, the survey data will be used to build a topological data analysis ${ }^{4}$ that creates student attitudinal profiles based on past and present STEM-related experiences. It will also highlight doctoral-level experiences that are related positively and negatively to attitudinal profile development. In Phase 3 (qualitative), a small sample of doctoral students from various attitudinal profiles 
identified in Phase 2 will be recruited for follow-up interviews. Through our mixed-methods approach, we will capture the complex interaction of students' identities and motivation and begin to unravel how students' academic experiences influence these attitudinal profiles.

\section{Completed Work}

Phase 1: During the Fall 2015 and Spring 2016 semesters, interview and focus group data were collected from two large universities with engineering colleges in the west and southeast. A pilot focus group with five students was conducted to test the interview protocols. In total, four interview protocols were developed, which sought to characterize student experiences with 1) future time perspective (FTP) development, ${ }^{2}$ 2) identity development, ${ }^{1} 3$ ) the role of identity based motivation (IBM) in graduate education, ${ }^{5}$ and an interview related to 4 ) graduate experiences. The first three interview protocols were developed to test the theoretical assumptions of this project while the last protocol was designed to be more open-ended and student led as to capture student experiences that may not have been outlined in the original proposal. We interviewed 41 engineering graduate students in focus groups and individual interviews using the four unique interview protocols. Students were selected from a range of engineering graduate programs to capture a wider breadth of experiences.

We employed interpretive phenomenological analysis (IPA) as a tool to analyze interview data. A team of four student researchers used IPA to explore how engineering students perceived their graduate experiences as related to each interview protocol. We also modified the methodological tradition of IPA to allow teaming across a research group as well as comparison between participants and frameworks. ${ }^{6}$

Phase 2: Following IPA analyses, we developed novel Likert-type survey measures of graduate student future time perspectives, identities, identity based motivations, and general experiences. We consulted with field experts in each of these areas for feedback on item development. In addition, we developed a statistically robust sampling plan based on data collected and protocol followed by the American Society for Engineering Education, which will be implemented during survey deployment in Spring 2017. The protocol stratifies degree programs by geographic location and samples degree programs from each stratum. This sampling is based on probability proportional to size for frequency of each engineering sub-discipline in the geographical setting. We have designated geographical location as the ultimate sampling unit and degree program as the primary sampling unit, with the intention of sampling each student within the primary sampling unit.

\section{Significant Results}

Results indicate that successful graduate experiences may be driven by students' perceived level of autonomy toward a variety of tasks, not just those purely centered in research, teaching, or service alone. These results highlight the importance of deliberately constructed mentoring experiences that allow students to develop their autonomy and competency so that they have the agency to act in their graduate programs of study. Initial results indicate that student perceptions of their graduate experiences are driven by a multi-faceted set of factors including: 
- their perceptions of task difficulty and their control over how and when difficulty is applied in order to inform the possibility and importance of those tasks. This is similar to trends found previously in the Identity-Based Motivation literature. ${ }^{5}$

- their perceived control over their experiences and actions, which directs how students value tasks and their desire to pursue those tasks again in the future. Specifically, these discussions were centered on teaching assistant experiences for early engineering graduate students. The idea of control mirrors previous discussions of autonomy found in the Self-Determination Theory literature. ${ }^{7}$

- their level of social integration into their communities of practice, including their relationships within their graduate/laboratory groups and advisors. This mirrors results indicating the significance of identity congruence to social context from the work of Oyserman and Destin. ${ }^{5}$

- the opportunity to integrate skills and knowledge from past and non-engineering experiences into current learning and research practices. The leveraging of past experiences for goal setting and identity integration expands previous results found in engineering identity literature. ${ }^{1}$

In-depth analysis of early results suggests that the development of a successful 'graduate student engineer' identity relies heavily on the integration of existing, deeply-entrenched identities and motivations. Without this integration, students' mental and emotional resources are divided and often pitted against each other, resulting in role conflict, disidentification, and withdrawal. This highlights the importance of environments that emphasize rich interconnections between personal, cultural, and engineering experiences and motivation so that students' identities will be consolidated rather than fragmented.

The results of this work also suggest that students have conceptualizations of their futures that do not match conversations traditionally seen in the STEM education literature, ${ }^{8}$ thus supporting the argument for testing and development of theories specific to graduate student populations. In contrast to previously interviewed undergraduate populations, the graduate students interviewed have an idea of their far future career in either an industrial or academic job. They also have contingency plans for their near future career, meaning they have different paths to reach their far future career based on what kind of job they can attain upon graduation. Graduate students also perceive instrumentality (usefulness) of their courses, ${ }^{9}$ as doing coursework helps them develop the skills they need to be an academic even when the content of their classes is not related to their dissertation research. While current results aggregate engineers across disciplines, further work is exploring if this assumption holds true.

\section{Future Work}

Future tasks and goals of the project involve the continued analysis of interview/focus group data from year one of the project using IPA methodology while also informing our quantitative instrument development as part of Phase 2. Our next major goal in Phase 2 is to gather evidence for reliability and validity of the instrument across different contexts. Specifically, we will work with content experts to develop face validity of the items and pilot the instrument using a test/retest model for reliability. Cognitive interviews will be used for content validity. Once the instrument has been developed and fully tested, we will recruit graduate programs across the US through employment of our sampling protocol. 


\section{Intended Impacts}

Our work in adapting advanced qualitative methodologies serves to advance ongoing conversations about quality in qualitative work and address pragmatic requirements of analyzing large amounts of data in robust ways. This moves beyond the conversation of developing a methodological outline to a guide of how IPA can be applied to large scale studies in engineering education.

Our work with graduate students' identities as engineers, students, and members of their peer and family groups has expanded the conversation about STEM identities by stepping outside of the traditional undergraduate classroom where these discussions are typically situated. By focusing on a population that is studying advanced STEM concepts and is in the process of crafting and enacting sophisticated and multi-faceted identities, we can learn more about how engineering identities develop when students are active, efficacious, and engaged; these are areas where traditional engineering programs are generally seeking to improve. At the same time, we can observe students as they begin negotiating their personal and professional responsibilities and thus can begin to determine when, where, and why students and early-career professionals struggle. By better understanding engineering graduate student development, we can explore ways to provide targeted and efficient support to graduate students and new professionals.

Our work in understanding engineering graduate student experiences provides useful tools for engineering education researchers and educators to begin understanding the experiences of this population. Specifically, utilizing the student perspective and moving beyond traditional institutional reporting begins to elucidate and provide evidence about the "true" engineering graduate experience. This increasingly accurate reflection of graduate experiences provides novel insight into the experiences of students that have been traditionally ignored or unjustifiably lumped in with other students who share the title of graduate student.

The initial findings of our qualitative analysis indicate that student perceptions of control and the ability to utilize multiple resources to overcome barriers are fundamental to the successful development of their identities and motivations. Students' perceptions of control provide a means of discerning the difficulty of a given choice or task, which informs the possibility and importance of accomplishing that choice or task. Many graduate programs have become complacent about their high attrition rates, stating that the students who are leaving are not qualified or ready to be graduate students. This work begins to outline the institutional features that may be forcing qualified students to make the difficult decision to leave their graduate programs. Our results point to the need for exploration of student relationships with multiple institutional agents including but not limited to their advisors, classmates, and lab mates.

\section{Acknowledgements}

This work is supported through funding by the U.S. National Science Foundation (EHR1535254). The authors wish to thank the participants of the study outlined in this work for their willingness to share their voices and experiences. 


\section{References}

1. Godwin A, Potvin G, Hazari Z, Lock R. Identity, critical agency, and engineering: an affective model for predicting engineering as a career choice. J. Eng. Educ.

2016;105(2):312-340.

2. Husman J, Lens W. The role of the future in student motivation. Educ Psychol 1999:113125.

3. Smith JA, Flowers P, Larkin M. Interpretative Phenomenological Analysis: Theory, Method and Research. SAGE Publications Ltd; 2009.

4. Nielson JL, Paquette J, Liu AW, et al. Topological data analysis for discovery in preclinical spinal cord injury and traumatic brain injury. Nat Commun 2015;6:8581.

5. Oyserman D, Destin M. Identity-based motivation: Implications for intervention. Couns Psychol 2010;38(7):1001-1043.

6. Kirn A, Godwin A, Cass C, Ross M, Huff J. Mindful Methodology: A transparent dialogue on adapting Interpretative Phenomenological Analysis for engineering education research. In: Mindful Methodology: A Transparent Dialogue on Adapting Interpretative Phenomenological Analysis for Engineering Education Research.; 2017.

7. Deci EL, Ryan RM. Self-determination theory: A macrotheory of human motivation, development, and health. Canadian Psychology/Psychologie canadienne 2008;49(3):182185 .

8. Nelson KG, Shell DF, Husman J, Fishman EJ, Soh L-K. Motivational and Self-Regulated Learning Profiles of Students Taking a Foundational Engineering Course. J. Eng. Educ. 2015;104(1):74-100.

9. Hilpert JC, Husman J, Stump GS, Kim W, Chung W-T, Duggan MA. Examining students' future time perspective: Pathways to knowledge building. Japanese Psychol Res 2012;54(3):229-240. 